\title{
Randomised controlled trial comparing narrative exposure therapy with present-centred therapy for older patients with post-traumatic stress disorder
}

J.C.G. Lely, J.W. Knipscheer, M. Moerbeek, F.J.J. ter Heide, J. van den Bout and R.J. Kleber

\section{Background}

Evidence-based treatment and age-specific services are required to address the needs of trauma-affected older populations. Narrative exposure therapy (NET) may present an appropriate treatment approach for this population since it provides prolonged exposure in a lifespan perspective. As yet, however, no trial on this intervention has been conducted with older adults from Western Europe.

\section{Aims \\ Examining the efficacy of NET in a sample of older adults.}

\section{Method}

Out-patients with post-traumatic stress disorder (PTSD), aged 55 years and over, were randomly assigned to either 11 sessions of NET ( $n=18)$ or 11 sessions of present-centred therapy (PCT) $(n=$ 15) and assessed on the Clinician-Administered PTSD Scale (CAPS) pre-treatment, post-treatment and at follow-up. Total scores as well as symptom scores (re-experience, avoidance and hyperarousal) were evaluated.

\section{Results}

Using a piecewise mixed-effects growth model, at post-treatment a medium between-treatment effect size for CAPS total score (Cohen's $d=0.44$ ) was found, favouring PCT. At follow-up, however, the between-treatment differences were non-significant. Drop-out rates were low (NET 6.7\%, PCT 14.3\%) and no participant dropped out of the study because of increased distress.

\section{Conclusions}

Both NET and PCT appear to be safe and efficacious treatments with older adults: PCT is non-intrusive and NET allows for imaginal exposure in a lifespan perspective. By selectively providing these approaches in clinical practice, patient matching can be optimised.

\section{Declaration of interest}

None.

\section{Keywords}

Post-traumatic stress disorder; narrative exposure therapy; present-centred therapy; older adults; randomised controlled trials.

\section{Copyright and usage}

(c) The Royal College of Psychiatrists 2019.
Globally, the number of older adults is increasing. ${ }^{1}$ Post-traumatic stress disorder (PTSD) $)^{2}$ in older adults is a serious condition, ${ }^{3}$ often associated with depression and somatic problems. Compared with younger cohorts, older adults present higher arousal and lower avoidance levels. ${ }^{4}$ Preliminary controlled trials on the efficacy of trauma-focused interventions for older adults - sample mean ages from 57 to 71 years ${ }^{5-8}$ - revealed mixed results for PTSD. The experimental interventions included narrative exposure therapy (NET), virtual reality exposure therapy and therapist-guided internetbased therapy; the controls included psychoeducation, wait lists and present-centred therapy (PCT), originally developed as Present Centered Therapy. Reported between-treatment effect sizes in Hedges' $g$ (from non-significance to 1.41) were substantially smaller than mean effect sizes of psychotherapy among adults in general: ${ }^{9}$ Hedges' $g=1.14,95 \%$ CI $[0.97 ; 1.3]$. The trial on $\mathrm{NET}^{5}$ yielded promising results, which call for replication. Because of barriers in assessment ${ }^{10}$ and clinical practice, ${ }^{11}$ adequate psychotherapeutic service for older people with PTSD is not self-evident. If traumatic backgrounds are not recognised in primary healthcare, older patients frequently get inappropriate treatments, leading to dissatisfaction and overconsumption of somatic care or medication. ${ }^{10}$ Furthermore, uncertainty exists about the need for traumafocused interventions when treating older people with $\mathrm{PTSD}^{12}$ and how to provide such treatments in an age-specific way. ${ }^{4}$

By embedding trauma-focused cognitive-behavioural therapy (TFCBT) in a lifespan perspective and by allowing for imaginal exposure of multiple traumatic memories, $\mathrm{NET}^{13}$ might be an appropriate treatment option for older adults. Medium to large effect sizes were found for NET for adults and good treatment results were found for older adults. ${ }^{14}$

In this randomised controlled trial (RCT), the efficacy of NET for older people with PTSD was compared with non-traumafocused PCT. ${ }^{15,16}$ In addition to PTSD severity (according to DSM-IV ${ }^{2}$ ) and diagnosis, individual treatment response and drop-out rates were reported. Consistent with the preliminary findings, ${ }^{5}$ a larger decline in PTSD symptoms was expected for NET participants than in the control group during treatment. During follow-up, it was expected that the differences between the trends of NET and PCT would be sustained. Finally, it was expected that mainly avoidance and hyperarousal symptoms would be reduced.

\section{Method}

Participants were recruited from two specialised Dutch centres (Foundation Centrum ' 45 and the Sinai Centrum) assessing and treating psychotrauma-related disturbances. After approval of the medical ethics committee at Leiden University (protocol number P13.009), participants were enrolled between April 2013 and April 2016. Eligible patients were approached by their intake therapists. After receiving a complete description of the study, those consenting gave written informed consent. To assess interrater reliability and treatment adherence, permission was asked to videotape all assessments and treatment sessions. Subsequently, those 
consenting were interviewed to formally check inclusion and exclusion criteria.

\section{Study entry criteria}

Participants were adults, aged 55 years or older, seeking individual treatment for PTSD in the trial sites. The limit of 55 years was set to capture important age-related challenges such as retirement, loss of family members and friends, and physical and cognitive changes. Eligibility required meeting DSM-IV-TR (2000) criteria for PTSD and, if taking psychotropic medication, maintaining a stable dose for at least 2 months. Participants were asked to keep their medication regimen unchanged throughout the treatment in consultation with their prescribers. Exclusion criteria involved severe cognitive impairment (Mini-Mental State Examination ${ }^{17}$ score $\leq 20$ ), current high suicide risk, active psychosis or bipolar disorder (assessed with the Mini-International Neuropsychiatric Interview), ${ }^{18}$ not meeting full PTSD-IV criteria on the ClinicianAdministered PTSD Scale (CAPS), ${ }^{19}$ current substance disorders and concurrent psychosocial treatment during the study. No restrictions were applied on language proficiency.

\section{Design}

This RCT compared NET with PCT. For ethical reasons, an active comparator was selected. PCT focuses on current problems, avoiding traumatic memories. Participants were randomly assigned to 11 sessions of NET or 11 sessions of PCT, using computer-generated random numbers. The senior researcher had access to the computer programme, kept a log file of all random assignments and assigned the participants to the therapists. To increase comparability, session length and number of both treatments were equated. Taking into account patients' preferences and availability, session frequency could vary from 1 to 2 weeks. This naturalistic approach resulted in highly variable assessment intervals.

\section{Interventions}

NET is a standardised, individual treatment intervention ${ }^{13}$ for PTSD which is based on TFCBT and includes components from testimonial therapy, ${ }^{20}$ such as the chronological narrative. Consequently, multiple adverse events can be processed. The intervention includes 4-15 sessions of $90 \mathrm{~min}$ each, depending on the number of traumatic experiences. In NET, therapist and patient collaboratively develop a chronological narrative of the patient's life, emphasising memories of trauma and perceived support. Developing and revising this autobiographical narrative allows the patient to re-experience avoided traumatic experiences in imaginal exposure. This procedure is considered to modify the patient's neural fear networks and to reorganise autobiographical memories, ${ }^{13}$ reducing symptoms and restoring narrative continuity. The narrative is written down and the resulting document may be used by the patient for legal or personal purposes. Therapists in both trial sites conducted NET following the manual. ${ }^{13}$ Session one is dedicated to psychoeducation and treatment planning. In session two, the therapist and the participant create a visual timeline of traumatic and supporting experiences and select the events for exposure. Sessions three to ten are dedicated to imaginal exposure. The last session allows for receiving the documented narration, focusing on the future and saying goodbye.

PCT, developed as a non-trauma-focused control condition, includes psychoeducation about PTSD and homework assignments targeting current maladaptive relational patterns by including problem-solving techniques, and helps patients to focus on the 'here and now'. Since PCT was found to show similar efficacy in reducing post-traumatic and depressive symptoms to that of trauma-focused psychotherapy, ${ }^{21,22}$ PCT could be presented as a credible therapeutic alternative. ${ }^{15}$ Moreover, PCT had significantly fewer participants dropping out than trauma-focused comparators. ${ }^{21}$ Therapists conducted PCT following the PCT protocol. ${ }^{15,16}$ After the introductory session, nine $90 \mathrm{~min}$ sessions are focused on relieving daily stress. In homework assignments, the patients select the relevant issues. The last session allows for summarising, looking forward and saying goodbye.

Both interventions involved registered psychotherapists, psychiatry residents or fully qualified psychosocial therapists with appropriate training in NET or PCT. All therapists had completed the required training; some of them had additional experience. If necessary, treatments were facilitated by professional interpreters over the telephone. To prevent investigator allegiance effects independent, qualified trainers and supervisors participated in the study. In supervision meetings, treatment content (including videotapes) and processes were evaluated. For both conditions, therapist manuals were designed, including study procedures and the medication protocol (manuals available on request). To systematically assess treatment content, a checklist (details available from the corresponding author on request) of prescribed or proscribed components was used, adapted for use in the interventions. To take account of the desired focus, the checklist was stratified (introduction, traumainformed psychoeducation and present-centred sessions), allowing for a detailed assessment. In PCT session reports, treatment content was summarised. Treatment adherence in the PCT was assessed by the assistant researchers or the senior researcher observing randomly selected videotaped treatment sessions (stratified by therapist and treatment phase). Additionally, all PCT session reports were screened for the abstinence of trauma exposure.

\section{Assessments}

PTSD-IV severity and diagnosis were measured using the CAPS $^{19}$ in Dutch translation. ${ }^{23}$ In addition to total scores, symptom cluster scores (re-experience, avoidance and arousal) were calculated. Finally, individual change and drop out were reported. The assessments were scheduled to take place pre-treatment, post-treatment and at follow-up (4 months). After follow-up assessment, participants converted to care as usual.

Assessments were conducted by trained, independent master students in clinical psychology with extensive training in the use of the CAPS, assisted by professional interpreters if necessary. As part of their training, all assessors signed a declaration of confidentiality and conducted diagnostic sessions under direct observation of the senior researcher, a registered psychotherapist. To ensure blinding of treatment allocation, assessors had limited access to participants' data; participants were asked not to reveal treatment content. All interpreters were trained for clinical interviews in psychotherapy and signed a declaration of confidentiality. After completing all assessments, participants received a gift coupon in appreciation of their time and effort.

The pre-treatment assessment included a structured interview about sociodemographic data. To assess traumatic experiences the CAPS Life Events Checklist was used. DSM-IV-TR (2000) diagnosis and symptom severity were assessed using the CAPS. The CAPS assesses frequency and intensity ratings (range $[0 ; 4]$ ) resulting in symptom scores (range $[0 ; 8]$ ) for all 17 PTSD symptoms according to DSM-IV-TR (total range $[0 ; 136]$ ). A symptom was considered present if its frequency was rated as at least one and its intensity as at least two. ${ }^{19}$ The CAPS has been widely used in trauma research, revealing strong psychometric properties. In previous studies, high internal consistency was reported: Cronbach's $\alpha=[0.89 ; 0.95] .{ }^{23-26}$ Good internal reliability was maintained in different translations. ${ }^{23,25}$ High specificity and sensitivity were found with older combat veterans. ${ }^{26}$ 


\section{Statistical analysis}

Power calculations were based on the assumption of small to medium relative effect sizes. Using $G^{\star}$ Power version 3.1 for Windows, ${ }^{27}$ a total sample size of 28 was estimated (assuming a power of 0.80 , a two-sided significance level of 0.05 and three repeated measures). To allow for participant attrition of $20 \%$, the aim was a final sample size of 34 .

Since the treatment and follow-up duration highly varied across participants, a piecewise mixed-effects growth model was used to determine weekly change rates in the four outcome measures across time (therapy versus follow-up) and treatments. The time factor was scaled at zero corresponding to the post-treatment measurement. This multilevel model enabled the comparison of mean NET and PCT outcome scores at post-test. Between-subjects variation for the duration of treatment and follow-up could be taken into account, ${ }^{28}$ as well as between-subjects variability at post-treatment. This model permitted varying change rates during treatment and follow-up ${ }^{29}$ but required reformulating the expectations into operational hypotheses:

(a) during treatment, the outcome change rate is different for either condition;

(b) during follow-up, the outcome change rate is different for either condition;

(c) for NET, the outcome change rate is equal during treatment and follow-up;

(d) for PCT, the outcome change rate is different during treatment and follow-up;

(e) at post-treatment, the two conditions have different outcomes.

Four covariates were included in the model: comorbid depression symptoms and childhood trauma were considered to have a negative impact on the effectiveness of psychological treatments, ${ }^{30,31}$ female gender was associated with higher treatment effects for PTSD ${ }^{9,32,33}$ and higher numbers of traumatic events were found to increase PTSD symptom severity. ${ }^{34,35}$

Treatment adherence, interrater reliability and demographic and clinical variables were analysed with SPSS version 23 for Windows (IBM, Armonk, NY). $\chi^{2}$-tests or Fisher's exact tests and independent $t$-tests were used to compare demographic and clinical characteristics between participants and refusers, treatment completers and those who dropped out, as well as between the treatment conditions. Interrater reliability was calculated by dividing the proportion of agreements between two observers by the probability for that proportion.

The data were converted to the software MLwiN version 3.02 for Windows ${ }^{36}$ with restricted maximum likelihood and robust standard errors. The hypotheses were submitted to contrast testing. ${ }^{37}$ Underlying model assumptions were evaluated and potential multicollinearity of covariates was assessed by means of variance inflation factors. Furthermore, the relation between the number of traumatic events and CAPS scores was assessed by means of scatterplots. Twosided tests were conducted with a significance level of $\alpha=0.05$.

To evaluate individual treatment response, clinically significant change (indicated as treatment response) was rated. Treatment response was defined as a symptom reduction of ten or more points on CAPS ${ }^{38,39}$ and outcomes dropping under the CAPS cutoff value of 40 scoring points. Harm was defined as an increase in symptoms by ten or more points followed by drop out. Treatment drop out indicated the percentage of participants prematurely terminating treatment.

\section{Clinical trial registration}

This clinical trial was registered with the Netherlands Trial Register (NTR), under reference number 3987, and NARCIS (Dutch
National Academic Research and Collaborations Information System), OND1352440.

\section{Results}

\section{Participants}

Figure 1 provides details of the participant flow through the trial. The diagram includes all patients who met inclusion criteria at intake, i.e. before being informed about the study and formally interviewed. In total, 67 patients were approached; 36 patients were assessed for eligibility. Reported reasons to decline participation were fear of increased stress due to assessments, or refusal to be randomised. Table 1 displays the baseline and clinical statistics of the two treatment conditions.

Because of external circumstances, one participant's treatment was exceptionally long; consequently, this participant's data were removed from all further analyses. At baseline, no significant differences across conditions were found in the covariates and other clinical and sociodemographic variables, except for the assistance of interpreters. Participants were civilian trauma survivors, referred by primary physicians or medical specialists. Reported traumatic events included persecution and political and domestic violence, including childhood abuse. The participants' mean age was 63.81 years and $75 \%$ were males. Most participants originated from the Netherlands (51.5\%) and the Middle East (36.4\%). All participants had encountered multiple traumatic events (mean 9.15, s.d. 3.76). A total of 12 participants (36.4\%) reported childhood trauma, implying trauma occurring between 5 and 12 years of age; 10 participants (30.3\%) reported sexual trauma. As shown in Table 1, no significant between-group differences for those variables were found. From the participants, $60.6 \%$ suffered from comorbid depression and $9.1 \%$ were assisted by interpreters.

\section{Attrition}

After being included in the study, three patients refused to participate before starting treatment. Refusal involved not accepting the allocated intervention, not wanting to participate in the required assessments and fearing increase in stress following exposure sessions. Three more participants left treatment prematurely (two of them refusing to continue the prescribed number of sessions and assessments; one not being able to do so due to altered personal circumstances). Completer and drop-out rates did not significantly differ across treatment conditions $(t(1)=0.45, P=0.501)$. The $t$ tests revealed no significant differences in age $(t(27)=1.51, P=$ $0.142)$ or pre-treatment symptom severity $(t(27)=-0.52, P=$ 0.604 ) between completers and drop-outs.

\section{Treatment adherence}

Most therapists provided either NET or PCT. Four therapists provided both NET and PCT. Videotaped treatment sessions (both interventions) of those therapists were included in the treatment adherence assessment. Of all treatment sessions, 39 (12.96\%) sessions ( 21 for NET and 18 for PCT) were randomly selected for independent protocol adherence scoring by two assessors. On a scale of 100 , the overall mean treatment adherence ranged from 87 for PCT (s.d. 14) to 89 for NET (s.d. 11), which is considered excellent. According to the assessors, on average $87-89 \%$ of the desired treatment protocol components were applied during the interventions. Inspection of PCT session reports confirmed that no deviations of the prescribed focus were detected. Treatment adherence scores did not significantly differ for NET and PCT $(t(37)=0.515, P=$ $0.609)$. 


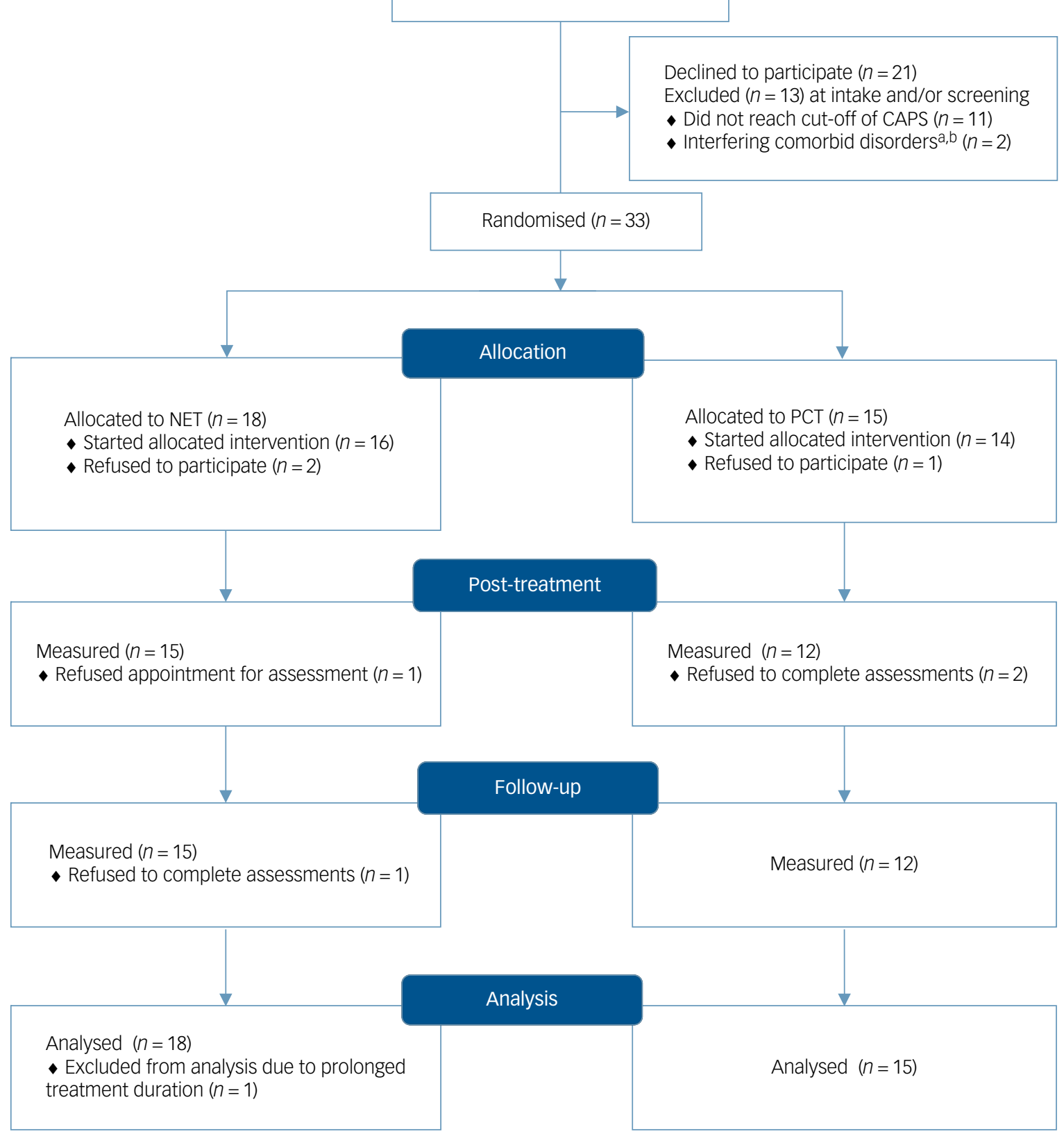

\section{Fig. 1 CONSORT 2010 flow diagram.}

\section{Reliability}

To assess the interrater reliability, $19.33 \%$ of all assessments were randomly elected, stratified for assessor, participant and time of assessment. A total of 14 independent assessors conducted the assessments; 7 of which were involved in assessing interrater reliability. Interrater reliability was good (Cohen's $\kappa=0.72$ ) for PTSD severity. Internal consistency for the CAPS was excellent (Cronbach's alpha $=0.88)$.

\section{Missing data}

From 396 scores (i.e. four outcome measures administered three times with 33 participants), 44 scores (11.1\%) were missing due to refusal after inclusion (7.1\%) or drop out (4\%).

\section{Outcomes}

Table 2 presents the mean outcomes and their s.d. at the three assessments per treatment group. Individual treatment response is presented in Supplementary Table 3 available at https://doi.org/10. 1192/bjp.2019.59 and shows the number of participants who reported recovery, treatment response, no response or symptom increase per treatment group.

No participant reporting increased symptoms left treatment prematurely. After starting treatment, one participant in the NET group developed dissociative symptoms and was admitted to residential care. The participant then continued NET according to the protocol as a single psychotherapeutic treatment.

During treatment, a majority of treatment completers in both conditions showed treatment response in PTSD severity. From 
Table 1 Baseline personal and trial characteristics of participants per treatment condition

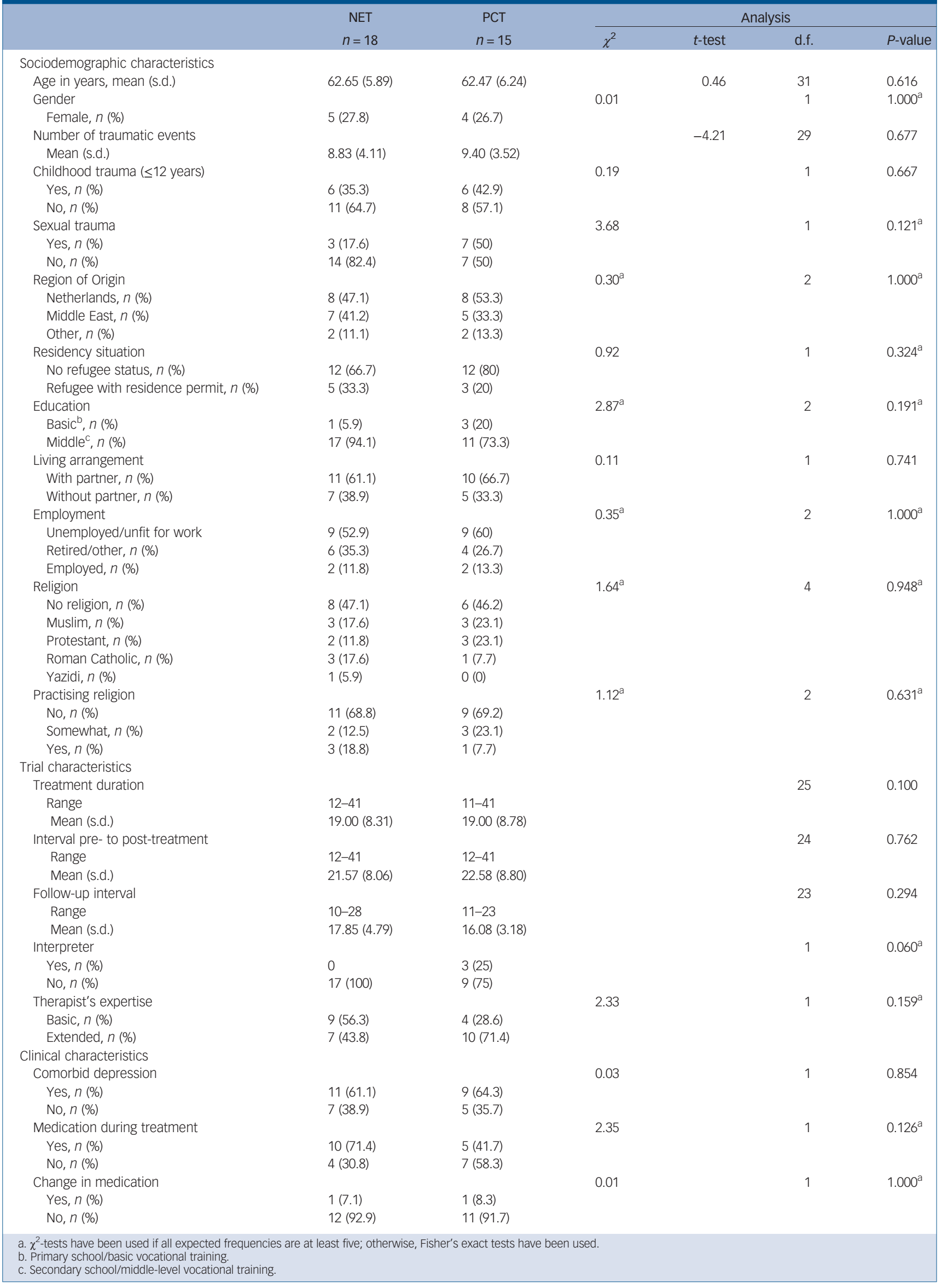


Table 2 Mean PTSD severity (CAPS) at baseline, post-treatment and follow-up, per treatment group

\begin{tabular}{|c|c|c|c|c|c|c|}
\hline \multirow[b]{2}{*}{ PTSD score } & \multicolumn{2}{|c|}{ Baseline } & \multicolumn{2}{|c|}{ Post-treatment } & \multicolumn{2}{|c|}{ Follow-up } \\
\hline & NET & PCT & NET & PCT & NET & PCT \\
\hline Total score, mean (s.d.) & $71.25(25.17)$ & $68.21(19.95)$ & $64.29(26.60)$ & $49.83(21.71)$ & $54.64(26.42)$ & $59.17(28.40)$ \\
\hline Re-experience, mean (s.d.) & $23.56(8.43)$ & $23.00(9.17)$ & $22.00(8.22)$ & 17.17 (9.33) & $19.00(9.04)$ & $20.50(8.61)$ \\
\hline Avoidance, mean (s.d.) & $25.06(12.46)$ & $23.86(11.74)$ & $21.21(12.74)$ & 17.14 (12.94) & $17.14(12.94)$ & $21.50(12.48)$ \\
\hline Arousal, mean (s.d.) & $23.25(7.00)$ & $21.36(4.98)$ & $21.07(7.51)$ & 17.58 (7.59) & $18.50(7.96)$ & $17.17(9.18)$ \\
\hline
\end{tabular}

pre-treatment to follow-up, $71.4 \%$ of the NET completers achieved a treatment response, compared with $50 \%$ of the PCT completers. During treatment, two NET completers (14.3\%) and two PCT completers (16.7\%) lost the diagnosis of PTSD. At follow-up, four NET completers $(28.6 \%)$ and one PCT completer $(8.3 \%)$ lost the diagnosis of PTSD. Figure 2 presents the outcomes, centred around posttreatment assessment.

Best model fit was obtained when including quadratic and cubic terms of the number of traumatic events. Scatterplots suggested the relation between the CAPS outcomes and the number of events was curvilinear. This relation was represented by a polynomial in the multilevel regression model and polynomials up to the fourth degree were examined. The significance of linear, quadratic cubic and quartic polynomial effects was tested by means of $t$-tests. The cubic term was the highest order polynomial term that was significant, hence third order polynomials were fitted. Scatterplots and quantile plots suggested that model assumptions of normality and homoscedasticity of residuals were met. Moreover, multicollinearity was irrelevant. The left part of each graph corresponds to the treatment phase, the right part to follow-up.

At post-treatment, significant between-group differences were found for two outcomes, favouring PCT. For total CAPS scores, the difference in mean scores amounted to 23.02 CAPS points (95\% CI [5.94; 40.42], $P=0.008$ ). For re-experience, the difference in mean scores was 9.25 points $(95 \%$ CI $[4.50 ; 14.29], P=0.000)$.

In the NET condition, a continuing decline for all outcomes was observed during treatment and follow-up. For PCT, a decline for all outcomes was only observed during treatment. During follow-up, PCT arousal scores continued to decrease over time; however, intrusion, avoidance and mean scores showed an increase and the PCT avoidance scores exceeded those in the NET condition. Supplementary Table 4 shows the $P$-values for the five hypothesis tests for each of the four outcomes.

For arousal symptoms, the weekly change rates did not differ significantly across treatments and time periods. For the other three outcomes, the weekly change rates during follow-up differed significantly across treatment conditions. Additionally, for these three outcomes, the weekly PCT change rates during treatment were significantly different from those during follow-up. For all outcomes, the weekly NET change rates during treatment did not differ significantly from those during follow-up. Post-treatment, there were significant betweentreatment differences in means for PTSD total score (Cohen's $d=$ $0.44)$ and re-experience symptoms $(d=0.40)$, which are considered medium effects. ${ }^{40}$ Finally, 17 weeks from post-treatment assessment (the mean follow-up interval), the between-group differences for all outcomes were non-significant. At this time point, for PTSD total score, re-experience and arousal symptoms, PCT still was favoured; but positions had reversed for avoidance symptoms.

\section{Discussion}

\section{Main findings}

From pre-treatment to post-treatment in this RCT, PCT showed a sharper decline than NET in PTSD symptoms for all symptom clusters. However, at follow-up the effects converged, showing a continuing symptom decline in the NET group and a partial PTSD symptom relapse in the PCT group. This relapse mainly involved symptoms of re-experience and avoidance. None of the participants in either condition left treatment prematurely because of an intolerable increase in symptoms.

\section{Efficacy and mechanisms}

The early symptom reduction in PCT can be understood by its nonintrusive character. Although intended to provide non-specific treatment factors, the current results support the use of PCT as an intervention with comparable effectiveness as trauma-focused psychotherapies in older adults. Traumatic memories are directly targeted during NET which increases stress levels, albeit temporarily. In the long run, the repetitive alternation of trauma exposure and cognitive elaboration (in the chronological narrative) is seen as effective memory processing, ${ }^{13}$ which is reflected in a continuing symptom decrease and increased cognitive coherence. The results for the NET group might imply that addressing re-experience and avoidance is required for a sustained treatment effect. The primary mechanisms of change from a present-centred perspective are based on altering maladaptive interpersonal communication. ${ }^{21}$ During follow-up, the partial symptom relapse for PCT participants may be due to chance, since the differences did not exceed the s.d. of the scores at post-test. However, the absence of treatment structure, with its homework and therapist support, may have played a role.

\section{Research in context}

The continued symptom reduction for NET in the current sample was consistent with other NET trials. ${ }^{14}$ The difference with other NET results in older adults ${ }^{5}$ might be explained by the highly active comparator, which was structurally equated and provided within high standard care. The non-significant between-treatment differences at follow-up are comparable with results of direct comparisons of trauma-focused therapies ${ }^{41-43}$ or the results of Eye Movement Desensitisation and Reprocessing (EMDR) compared with stabilisation. ${ }^{44}$ The PCT results were consistent with PCT research, ${ }^{8,21,22}$ underscoring the importance of the non-specific treatment factors and contesting the current PTSD treatment guidelines which prefer trauma-focused therapy over non-traumafocused alternatives. ${ }^{45}$

\section{Treating PTSD in older adults}

PTSD treatments for older adults evoke questions about the safety of trauma processing for vulnerable people and the need of trauma exposure to effectively treat them. ${ }^{11}$ Directly targeting traumatic memories in NET did not harm these vulnerable participants. Treatment drop-out rates were low in comparison to those in other trials involving PTSD treatments. ${ }^{46}$

The current findings support evidence that both PCT and NET are feasible interventions for older adults. In this population, PTSD and other health problems, decreasing autonomy or social isolation 

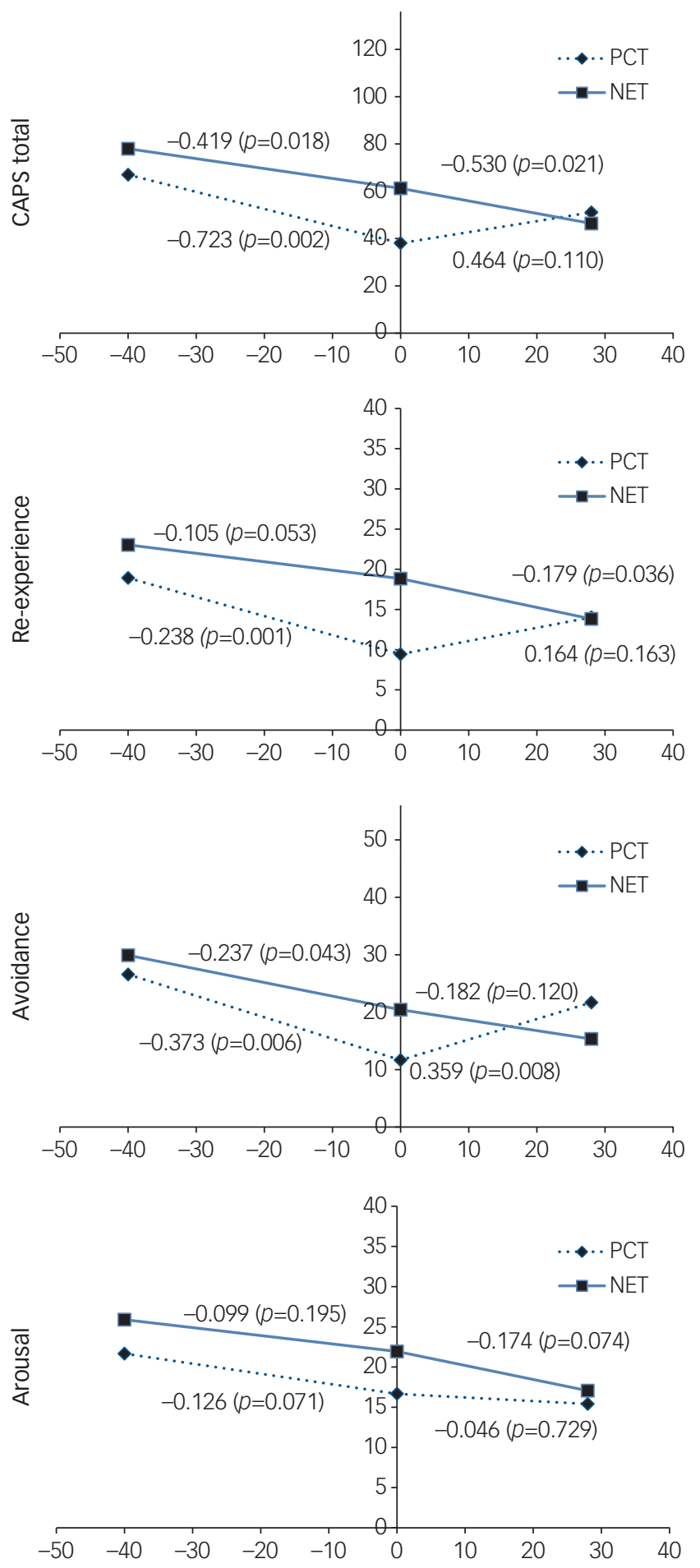

Fig. 2 Outcomes of CAPS total, re-experience, avoidance and arousal scores. PCT, present-centred therapy; NET, narrative exposure therapy.

The graphs represent females without depression and childhood trauma and report the mean number of traumatic events. Graphs for other covariate values show the same pattern, albeit shifted along the vertical axis. In the graphs, $P$-values indicate the significance of weekly change rates.

can burden coping mechanisms. From a patient's viewpoint, PCT can be regarded as an attractive treatment approach as it addresses current relational problems. Exposure therapy is much more taxing and symptoms take longer to abate. However, many older adults prefer to share their experiences, ${ }^{46}$ with the aim of re-appraising maladaptive meanings and with the hope to break the cycles of re-experience and avoidance, which frequently maintain exhaustion and despair. ${ }^{46}$

\section{Strengths and limitations}

The current study offers a controlled comparison of two treatment approaches for older survivors of traumatic events. A broad range of older people was engaged in this study, some needing interpreters. To enhance external validity, treatment duration was adapted to the patients' preferences and availability. The resulting variability of inter-assessment intervals was addressed by advanced statistical analyses. This approach allowed for significance testing of posttreatment effects, comparing the change rates across participants and full intention-to-treat analysis.

Several limitations must be considered. Since the participants may have been less avoidant than the refusers, the sample may suffer from (self-)selection bias. The uneven distribution of males and females might present additional selection bias. A more detailed control for therapist confounding would have been useful. Therapists' experience, however, was not related to differences between the treatment groups and careful assessment of treatment adherence showed that the risk of bias was low. Although intended, complete blindness could not be guaranteed, which is not unusual in psychotherapy trials. ${ }^{47}$ Additionally, the inclusion of covariates narrowed the analysis' external validity. The restricted participants' age range implies that the current example can be considered mainly representative for the younger cohorts of older adulthood. The flexible session frequency complicated an easy grasp of the results. Finally, the short mean follow-up interval may be considered a limitation; the converging results call for future research.

\section{Future research}

To generalise the current results, larger comparative studies with longer follow-up intervals and older participants are needed. To gain more insight into treatment components, comparisons of different treatment doses and/or session intervals might be useful, as well as comparing NET and/or PCT with other trauma-focused interventions (cognitive processing therapy, prolonged exposure, TFCBT, EMDR or brief eclectic psychotherapy for PTSD). Finally, validation of psycho-diagnostic instruments and re-evaluating cutoff scores for older adults will improve trauma-related care for this population.

\section{Clinical implications}

Trauma-affected older populations need accurate assessment and evidence-based treatment. This study suggests that both NET and PCT are effective and safe psychotherapeutic methods for PTSD treatment in older adults. The availability of different approaches may optimise treatment matching. If, for instance, post-treatment NET evaluation would reveal remaining relational problems, an alternative intervention targeting those issues, such as PCT but also Skills Training in Affect and Interpersonal Regulation, can serve as a subsequent treatment option. The opposite is equally possible: starting with current problems and later continuing with narrative exposure. The results of PCT underscore the impact of interpersonal problems in PTSD and offer a possible option for lifting this burden.

The early symptom reduction in PCT may cause people to prefer this treatment method. NET, however, should be the treatment of choice if patients prefer sharing their trauma story and adapting its meaning in their lives. Patient and therapist preferences have been shown to play an important role in the choice of 
treatment method; ${ }^{48,49}$ providing balanced information fosters realistic expectations and hence better results.

J.C.G. Lely, MSC (D), Psychotherapist and Researcher, Foundation Centrum '45; and Ara Psychotrauma Expert Group, the Netherlands; J.W. Knipscheer, PhD, Psychotherapist, Foundation Centrum '45; Arq Psychotrauma Expert Group; and Assistant Professor, Department of Clinical Psychology, Utrecht University, the Netherlands; M. Moerbeek, the Netherlands; F.J.J. ter Heide, PhD, MPhil Centrum '45; and Senior Researcher, Arq Psychotrauma Expert Group, the Netherlands J. van den Bout, PhD, Professor Emeritus, Department of Clinical Psychology, Utrech University, the Netherlands; R.J. Kleber, PhD, Professor Emeritus Psychotraumatology, Department of Clinical Psychology, Utrecht University; and Arq Psychotrauma Expert Group, the Netherlands

Correspondence: J.C.G. Lely, Foundation Centrum '45/Arq Psychotrauma Expert Group, Nienoord 13, 1112 XE Diemen, the Netherlands. Email: j.lely@centrum45.n

First received 22 Jun 2018, final revision 14 Dec 2018, accepted 5 Feb 2019

\section{Supplementary material}

Supplementary material is available online at https://doi.org/10.1192/bjp.2019.59.

\section{Funding}

This study was jointly funded by Fonds Nuts Ohra, a Dutch foundation for research and prometion on behalf of vulnerable groups in society, and Foundation Centrum '45, partner in Arq Psychotrauma Expert Group.

\section{Acknowledgements}

The authors thank the patients, the colleagues at Centrum ' 45 and the Sinai Centre, the junior researchers, research assistants and the library staff who contributed to this study.

\section{References}

1 United Nations (UN) Department of Economic and Social Affairs. World Population Ageing, 2017. UN, 2017 (http://www.un.org/en/development/ desa/population/publications/pdf/ageing/WPA2017_Highlights.pdf)

2 American Psychiatric Association (APA). Diagnostic and Statistical Manual of Mental Disorders (4th edn, Text Revision). APA, 2000

3 Van Zelst WH, De Beurs E, Beekman ATF, Deeg DJH, Van Dyck R. Prevalence and risk factors of posttraumatic stress disorder in older adults. Psychother Psychosom 2003; 72: 333-42.

4 Böttche M, Kuwert P, Knaevelsrud C. Posttraumatic stress disorder in older adults: an overview of characteristics and treatment approaches. Int J Geriatr Psychiatry 2012; 27: 230-39.

5 Bichescu D, Neuner F, Schauer M, Elbert T. Narrative exposure therapy fo political imprisonment-related chronic posttraumatic stress disorder and depression. Behav Res Ther 2007; 45: 2212-20.

6 Gamito P, Oliveira J, Rosa P, Morais D, Duarte N, Oliveira S, et al. PTSD elderly war veterans: a clinical controlled pilot study. Cyberpsychol Behav Soc Netw 2010; 13: 43-8.

7 Knaevelsrud C, Böttche M, Pietrzak RH, Freyberg HJ. Efficacy and feasibility of a therapist-guided internet-based intervention for older persons with childhood traumatization: a randomized controlled trial. Am J Geriatr Psychiatry 2017; 25: 878-88.

8 Ready DJ, Gerardi RJ, Backschneider AG, Mascaro N, Olasov Rothbaum B. Comparing virtual reality exposure therapy to present-centered therapy with 11 U.S. vietnam veterans with PTSD. Cyberpsychol Behav Soc Netw 2010; 13: 49-54.

9 Watts BV, Schnurr PP, Mayo L, Young-Xu Y, Weeks WB, Friedman MJ. Metaanalysis of the efficacy of treatments for posttraumatic stress disorder. J Clin Psychiatry 2013; 74: e541-50.

10 Van Zelst WH, De Beurs E, Beekman ATF, Van Dijck R, Deeg DJH. Well-being physical functioning, and use of health services in the elderly with PTSD and subthreshold PTSD. Int J Geriatr Psychiatry 2006; 21: 180-88.

11 Arean P. Implementing evidence-based psychotherapies in settings serving older adults: challenges and solutions. Psychiatr Serv 2012; 63: 605-7.

12 Thorp SR, Wells SY, Cook JM. Trauma focused therapy for older adults. In APA Handbook of Trauma Psychology; Vol 2. Trauma Practice 2017 (ed SN Gold). American Psychological Association, 2017.
13 Schauer M, Neuner F, Elbert T. Narrative Exposure Therapy. A Short-term Intervention for Traumatic Stress Disorders after War, Terror or Torture (2nd, expanded edition). Hogrefe \& Huber Publishers, 2011.

14 Robjant $\mathrm{K}$, Fazel $\mathrm{M}$. The emerging evidence for narrative exposure therapy: A review. Clin Psychol Rev 2010; 30: 1030-9.

15 McDonagh A, McHugo G, Sengupta A, Demment C, Schnurr P, Friedman M, et al. Randomized trial of cognitive-behavioral therapy for chronic posttraumatic stress disorder in adult female survivors of childhood sexual abuse. J Consult Clin Psychol 2005; 73: 515-24.

16 Schnurr PP, Friedman MJ, Engel CC, Foa EB, Shea MT, Chow BK, et al. Cognitive behavioural therapy for posttraumatic stress disorder in women: a randomized controlled trial. JAMA 2007; 297: 820-30.

17 Folstein MF, Folstein SE, McHugh PR. "Mini-mental State". A practical method for grading the cognitive state of patients for the clinician. J Psychiatr Res 1975; 12: 189-98

18 Sheehan DV, Lecrubier $\mathrm{Y}$, Sheehan $\mathrm{KH}$, Amorim $\mathrm{P}$, Janavs J, Weiller E, et al. The MINI International Neuropsychiatric Interview (M.I.N.I.): the development and validation of a structured diagnostic psychiatric interview for DSM-IV and ICD10. J Clin Psychiatry 1998; 59: 22-33.

19 Blake DD, Weathers FW, Nagy LM, Kaloupek DG, Gusman FD, Charney DS, et al. The development of a clinician-administered PTSD scale. J Trauma Stress 1995; 8: 75-90.

20 Cienfuegos J, Monelli C. The testimony of political repression as a therapeutic instrument. Am J Orthopsychiatry 1983; 53: 43-51.

21 Frost ND, Laska KM, Wampold BE. The evidence for present-centered therapy as a treatment for posttraumatic stress disorder. J Trauma Stress 2014; 27: $1-8$

22 Steenkamp MM, Litz BT, Hoge CW, Marmar CR. Psychotherapy for military-related PTSD. A review of randomized clinical trials. JAMA 2015; 314: 489-500.

23 Hovens JE, Van der Ploeg HM, Klaarenbeek MTA, Bramsen I, Schreuder JN, Vladar Rivero V. The assessment of PTSD with the clinician administered PTSD scale: Dutch results. J Clin Psychol 1994; 50: 325-40.

24 Weathers FW, Ruscio AM, Keane TM. Psychometric properties of nine scoring rules for the Clinician-Administered Posttraumatic Stress Disorder Scale. Psychol. Assess. 1991; 11: 124-33.

25 Hinton DE, Chhean D, Pich F, Pollack MH, Orr SP, Pitman RK. Assessment of posttraumatic stress disorder in Cambodian refugees using the ClinicianAdministered PTSD Scale: psychometric properties and symptom severity. $J$ Trauma Stress 2006; 19: 405-9.

26 Hyer L, Summers MN, Boyd S, Litaker M, Boudewyns P. Assessment of older combat veterans with the Clinician-Administered PTSD Scale. J Trauma Stress 1996; 9: 587-93.

27 Faul F, Erdfelder E, Lang A-G, Buchner A. G*Power 3: A flexible statistical power analysis program for the social, behavioral, and biomedical sciences. Behav Res Methods 2007; 39: 175-91.

28 Naumova EN, Must A, Laird NM. Tutorial in Biostatistics: evaluating the impact of critical periods' in longitudinal studies of growth using piecewise mixed models. Int J Epidemiol 2001; 30: 1332-41.

29 Hox J, Moerbeek M, Van de Schoot R. Multilevel analysis. Techniques and Applications (3rd edn). Routledge, 2018.

30 Dunn E, Nishimi K, Powers A, Bradley B. Is developmental timing of trauma exposure associated with depressive and post-traumatic stress disorder symptoms in adulthood? J Psychiatr Res 2017; 84: 119-27.

31 Rytwinski NK, Scur MD, Feeny NC, Youngstrom EA. The co-occurrence of major depressive disorder among individuals with posttraumatic stress disorder: a meta-analysis. J Trauma Stress 2013; 26: 299-309.

32 Stenmark H, Guzey IC, Elbert T, Holen A. Gender and offender status predicting treatment success in refugees and asylum seekers with PTSD. Eur J Psychotraumatol 2014; 5: 20803.

33 Tarrier N, Sommerfield C, Pilgrim H, Faragher B. Factors associated with outcome of cognitive-behavioural treatment of chronic post-traumatic stress disorder. Behav Res Ther 2000; 38: 191-202.

34 Kolassa IT, Ertl V, Eckart V, Kolassa S, Onyut LP, Elbert T. Spontaneous remission from PTSD depends on the number of traumatic event types experienced. Psychol Trauma 2010; 2: 169-74.

35 Ogle CM, Rubin DC, Siegler IC. Cumulative exposure to traumatic events in older adults. Aging Ment Health 2014; 18: 316-25.

36 Rasbash J, Steele F, Browne WJ, Goldstein H. A User's Guide to MLwiN, Version 2.32. Centre for Multilevel Modelling, 2015 (http://ow.ly/WbLE308upOp).

37 Goldstein H. Multilevel Analysis (4th edn). Wiley, 2011.

38 Schnurr PP, Friedman MJ, Foy DW, Shea MT, Hsieh FY, Lavort PW, et al. A randomized trial of trauma focus group therapy for posttraumatic stress disorder: results from a Department of Veterans Affairs Cooperative Study. Arch Gen Psychiatry 2003; 60: 481-9. 
39 Schnurr PP, Lunney CA. Symptom benchmarks of improved quality of life in PTSD. J Depress Anxiety 2016; 33: 247-55.

40 Cohen J. Quantitative methods in psychology. A power primer. Psychol Bull 1992; 112: 155-95.

41 Nijdam MJ, Gersons BPR, Reitsma JB, De Jongh A, Olff M. Brief eclectic psychotherapy v. eye movement desensitisation and reprocessing therapy for post-traumatic stress disorder: randomised controlled trial. Br J Psychiatry 2012; 200: 224-31.

42 Sloan DM, Marx BP, Lee DJ, Resick PA. A brief exposure-based treatment vs cognitive processing therapy for posttraumatic stress disorder: A randomized noninferiority clinical trial. JAMA Psychiatry 2018; 75: 233-9.

43 Tran US, Gregor B. The relative efficacy of bona fide psychotherapies for posttraumatic stress disorder: A meta-analytical evaluation of randomized controlled trials. BMC Psychiatry 2016; 16: 266

44 Ter Heide FJJ, Mooren TM, Van de Schoot R, De Jongh A, Kleber RJ. Eye movement desensitisation and reprocessing therapy v. stabilisation as usual for refugees: randomised controlled trial. Br J Psychiatry 2016; 209 $311-8$
45 Imel ZE، Laska K, Jakupcak M, Simpson TL. Meta-analysis of dropout in treatments for posttraumatic stress disorder. J Consult Clin Psychol 2013; 81: 394-404.

46 Curran B, Collier E. Growing older with post-traumatic stress disorder. J Psychiatr Ment Health Nurs 2016; 23: 236-42.

47 Bisson Jl, Roberts NP, Andrew M, Cooper R, Lewis C (2013) Psychological therapies for chronic post-traumatic stress disorder (PTSD) in adults. Cochrane Database Syst Rev. Dec 13, (12): CD003388 (doi: 10.1002/14651858.CD003388. pub4).

48 Van Minnen A, Hendriks L, Olff M. When do trauma experts choose exposure therapy for PTSD patients? A controlled study of therapist and patient factors. Behav Res Ther 2010; 48: 312-20. doi: 10.1016/j.brat.2009.12.003.

49 Simiola V, Neilson EC, Thompson R, Cook JM. Preferences for trauma treatment: A systematic review of the empirical literature. Psychol Trauma 2015; 7: $516-24$ 